\title{
Direct action of an angiotensin II receptor blocker on angiotensin II-induced left atrial conduction delay in spontaneously hypertensive rats
}

\author{
Narihisa Matsuyama ${ }^{1}$, Takeshi Tsutsumi ${ }^{2}$, Nami Kubota ${ }^{3}$, Toshiaki Nakajima ${ }^{3}$, Hiroshi Suzuki ${ }^{4}$ and \\ Youici Takeyama ${ }^{4}$
}

In this study, the inhibitory action of angiotensin II (Ang II) receptor blocker (ARB) on Ang II-induced conduction blocking in the left atrium was studied. Transmembrane action potentials and effective refractory period (ERP) were recorded from the left atrial trabeculae of spontaneously hypertensive rats $\left(\mathrm{SHR}_{\mathrm{Izm}} \mathrm{s}\right)$ that had been treated with or without olmesartan medoxomil (AT1 receptor blocker, $3.0 \mathrm{mg} \mathrm{kg}^{-1}$ ) for 8 weeks before the recording. During rapid electrical stimulation (RES), changes in activation time (AT) and frequencies of the conduction block were studied under perfusion of Ang II $\left(10^{-8} \mathrm{M}\right)$ or Ang II plus ARB $\left(10^{-7} \mathrm{M}\right)$. Interstitial fibrosis in the left atrium was quantitatively assessed using histological sections. Although action potential parameters and ERP did not differ between the two groups when preparations were driven with a basic cycle length, an Ang II-induced conduction delay was observed during RES, and this was significantly suppressed in $\mathrm{SHR}_{\mathrm{Izm}} \mathrm{S}$ treated with ARB $(P<0.05)$. The Ang II-induced conduction block was also significantly reduced with perfusion of ARB $(P<0.05)$. The interstitial fibrous area was significantly larger in untreated $\mathrm{SHR}_{\mathrm{Izm}} \mathrm{S}$ than in treated animals $(P<0.01)$. This antagonizing action of AT1 blockade might contribute to its non-antiarrhythmic therapeutic effect on atrial fibrillation because of its indirect and direct actions on the structural and functional remodeling of the left atrium.

Hypertension Research (2009) 32, 721-726; doi:10.1038/hr.2009.89; published online 10 July 2009

Keywords: angiotensin II type 1 receptor; atrial action potential; atrial fibrillation; conduction block; reverse remodeling

\section{INTRODUCTION}

Recent meta-analyses have shown that an angiotensin-converting enzyme inhibitor and an angiotensin II (Ang II) receptor blocker (ARB) are both effective at preventing new and recurrent atrial fibrillation $(\mathrm{AF}) .{ }^{1,2} \mathrm{~A}$ plausible pathophysiological mechanism for AF prevention may be the indirect antiarrhythmic actions of these drugs, including reverse atrial remodeling, attenuation of left atrial (LA) stretch and inflammation. ${ }^{2-5}$ However, the cellular electrophysiological actions induced by these drugs in normal LA tissue and LA tissue remodeled because of spontaneous hypertension are not known. If Ang II blockade is beneficial for AF prevention, as shown in clinical studies, any direct cellular electrophysiological actions induced by these drugs in LA tissue should be examined. Therefore, in this study, LA preparations from spontaneously hypertensive rats $\left(\mathrm{SHR}_{\mathrm{Izm}} \mathrm{s}\right)$ were dissected. Transmembrane action potentials and activation times (ATs) were measured during rapid electrical stimulation (RES). Using this model, the actions of Ang II on impulse propagation through LA trabeculae were evaluated in male adult $\mathrm{SHR}_{\mathrm{Izm}} \mathrm{s}$ treated with and without the ARB, olmesartan.

\section{METHODS}

Animals and preparations

Adult male SHR $\mathrm{Izm}_{\mathrm{s}} \mathrm{s}(n=24)$, obtained from the Disease Model Cooperation Research Association (Tokyo, Japan), were used to investigate remodelingassociated electrophysiological changes. To evaluate the effects of reverse remodeling, an Ang II type 1 receptor blocker (AT1 blocker; olmesartan medoxomil $3.0 \mathrm{mg} \mathrm{kg}^{-1}$ per day; Daiichi Sankyo Co. Ltd, Tokyo, Japan) was orally administered $(n=12)$ for 8 weeks from 14 to 22 weeks of age. ${ }^{6}$ Experimental procedures were conducted in accordance with the National Institutes of Health guidelines, and were approved by the Showa University Institutional Animal Care and Use Committee.

After body weight and systolic arterial blood pressure (using the tail-cuff method) were measured, each rat was anesthetized with sodium pentobarbital $\left(50 \mathrm{mg} \mathrm{kg}^{-1}\right.$, intra-peritoneal injection), and its heart was quickly removed. The left atrium with the atrial septum was dissected from the mitral orifice. The preparation was mounted in a lucite superfusion chamber in which the effective volume was $2.5 \mathrm{ml}$. The LA preparation was attached by its epicardial surface to the bottom of the chamber.

The LA endocardial surface could be seen when the cut edges were pinned (Figure 1a). The preparations were superfused with oxygenated normal Tyrode solution $\left(3 \mathrm{ml} \mathrm{min}^{-1}\right.$ ) bubbled with $97 \% \mathrm{O}_{2}$ and $3 \% \mathrm{CO}_{2}$ at a temperature of

\footnotetext{
${ }^{1}$ Department of Cardiology, Yamato Municipal Hospital, Yamato, Kanagawa, Japan; ${ }^{2}$ Department of Internal Medicine, Eda Memorial Hospital, Yokohama, Kanagawa, Japan; ${ }^{3}$ Department of Ischemic Circulatory Physiology, University of Tokyo, Bunkyo-Ku, Tokyo, Japan and ${ }^{4}$ Department of Cardiology, Showa University Fujigaoka Hospital, Yokohama, Kanagawa, Japan

Correspondence: Dr N Matsuyama, Department of Cardiology, Yamato municipal Hospital, 8-3-6 Fukaminishi, Yamato 242-8602, Japan.

E-mail: yamamoto-clinic@jcom.home.ne.jp
}

Received 8 January 2009; revised 11 May 2009; accepted 13 May 2009; published online 10 July 2009 



Figure 1 Left atrial preparation and experimental setup. The photograph in (a) shows the left atrial (LA) preparation pinned to the bottom of the superfusion chamber. (b) A representative transmembrane action potential recorded from an LA myocardial cell. A schematic of the LA preparation and experimental setup is illustrated in (c). The thick trabeculae traversed from the mitral orifice (MO) to the LA appendage (App), which is characteristic of male adult $\mathrm{SHR}_{\mathrm{Izm}} \mathrm{S}$. The electrical stimulation was applied to the root of a trabecula originating from the mitral orifice. An enlarged schematic of the tip of the modified bipolar stimulating electrodes is shown in the square frame to the right of (c). Arrows indicate the direction of current flow. $E_{1}$ and $E_{2}$, recording glass microelectrodes; St.E., modified bipolar stimulating electrodes; MO, mitral orifice; App, left atrial appendage; SHR, spontaneously hypertensive rat. A full color version of this figure is available at the Hypertension Research journal online.

$37 \pm 0.2{ }^{\circ} \mathrm{C}$. The composition of the Tyrode solution was as follows (mM): $\mathrm{NaCl}$ 137.0; $\mathrm{NaH}_{2} \mathrm{PO}_{4} 1.8 ; \mathrm{NaHCO}_{3} 12.0 ; \mathrm{MgCl}_{2}$ 0.5; $\mathrm{KCl}$ 5.4; $\mathrm{CaCl}_{2}$ 1.8; glucose 5.5; $\mathrm{pH}$ adjusted to $7.2-7.3$. Equilibration was allowed for at least $30 \mathrm{~min}$ to obtain stable recordings.

The preparation was driven by electrical current stimulation at $2 \mathrm{~Hz}$ applied at the end of the LA preparation near the mitral orifice. The intensity of the current pulse was twice that of the threshold, and the pulse duration was $2 \mathrm{~ms}$. The pulses were delivered to the preparation with modified bipolar electrodes (Figure 1c). To pass flat-shaped excitation fronts through the surface of the trabecula, modified bipolar electrodes were used. The expanded tip of a modified concentric circle bipolar electrode is illustrated in the square inset of Figure 1c. When the internal core of this electrode is charged and the cathode contacts the surface of the specimen, the impulse propagation stabilizes and AT can be measured even from small specimens like rat LA trabeculae. AT was defined as the interval from the stimulation artifact to the peak of $\mathrm{d} V / \mathrm{d} t_{\max }$. To measure transmembrane action potential and AT, glass microelectrodes filled with $3 \mathrm{M} \mathrm{KCl}$ and with a tip resistance of $20 \pm 5 \mathrm{M} \Omega$ were used. These were connected to a high-impedance DC amplifier with input capacity neutralization (MEZ 3000; Nihon Kohden Co. Ltd, Tokyo, Japan). As shown in Figure 1c, one microelectrode $\left(\mathrm{E}_{1}\right)$ was embedded in a myocardial cell $3 \mathrm{~mm}$ away from the stimulation site. The action potential was recorded as the voltage difference between the recording microelectrode and the other neutral microelectrode, which was placed in the perfusate as close as possible to the recording microelectrode $\left(\mathrm{E}_{2}\right)$. The effective refractory period (ERP) was measured as the minimum coupling interval eliciting a conductive action potential by premature stimulation applied to the preparations every eight basic stimuli. The action potential and the maximum upstroke velocity of the action potential $\left(\mathrm{d} V / \mathrm{d} t_{\max }\right)$ were displayed on the oscilloscope screen and captured as animations (frame rate, $33 \mathrm{~s}^{-1}$ ) with a DVD recorder (DZ-MV580 WOOO; Hitachi Co. Ltd, Tokyo, Japan) or photographed with a polaroid camera (Figure 1b). At the same time, these signals were simultaneously recorded on a strip chart using photosensitive paper (RTA-1100; Nihon Kohden Co. Ltd).

\section{Experimental protocol}

After the ERP, action potentials and $\mathrm{d} V / \mathrm{d} t_{\max }$ were measured as baseline recordings. The changes in action potential parameters (action potential duration, APD; resting membrane potential, RMP; $\mathrm{d} V / \mathrm{d} t_{\max }$ ) under the superfusion of Ang II ( $10^{-8} \mathrm{M}$; Sigma Chemical Co., St Louis, MO, USA) or Ang II plus the AT1 blocker (olmesartan; $10^{-7} \mathrm{M}$ ) were measured in the two groups: adult $\mathrm{SHR}_{\mathrm{Izm}} \mathrm{S}(n=12)$ and adult $\mathrm{SHR}_{\mathrm{Izm}} \mathrm{s}$ treated with olmesartan during the previous 8 weeks $(n=12)$. $\mathrm{WKY}_{\text {Izm }}$ rats $(n=8)$ were used as control to determine whether Ang II affects the action potential parameters and AT of the left atrium. Subsequently, the same recordings were performed during RES lasting $60 \mathrm{~s}$. The cycle length of the RES was shortened by $10 \mathrm{~ms}$ until a 2:1 conduction block appeared. The cycle length that was $20 \mathrm{~ms}$ longer than what was required to induce a 2:1 conduction blocking was defined as the cycle length of RES. The AT and the frequency of the conduction block were measured during RES for $60 \mathrm{~s}$ and compared between the experimental groups.

\section{Histological examination and evaluation of fibrous tissue}

The left atria were isolated from rat hearts and fixed overnight in buffered neutral formalin. Serial paraffin-embedded tissue sections were stained with hematoxylin-eosin and Masson's trichrome. Microscopic images were saved into a personal computer as picture files. Using Scion Image software (Scion, Frederick, MD, USA), interstitial fibrous tissue stained with blue color was quantitatively assessed by the brightness of its color and expressed as a percentage of the unit area $\left(100 \times 100 \mu \mathrm{m}^{2}\right)$.

\section{Statistical analysis}

All measurements are shown as mean \pm s.e.m. The statistical significance of intergroup differences in quantitative data was evaluated using Student's $t$-test (two tailed, unpaired). A value of $P<0.05$ was considered statistically significant. 


\section{RESULTS}

Table 1 shows the changes in body weight, systolic arterial blood pressure and heart rate, as well as the parameters measured from the LA action potential and the ERP when the preparation was driven with basic cycle length $(2 \mathrm{~Hz})$. Data were compared between olmesartan-treated and non-treated preparations. The systolic blood pressure was significantly lower in the olmesartan group, but there were no significant differences in body weight, heart rate, RMP, $A P D_{75}, d V /$ $\mathrm{d} t_{\max }$ or ERP between the two groups. The changes in LA action potentials and $\mathrm{d} V / \mathrm{d} t_{\max }$ at $0,20,40$ and $60 \mathrm{~s}$ after RES are shown in Figure 2. Panels a-1 and a-2 show the LA action potentials recorded from $\mathrm{WKY}_{\mathrm{Izm}} \mathrm{s}$, and panels $\mathrm{b}-1$ and $\mathrm{b}-2$ show recordings from untreated $\mathrm{SHR}_{\mathrm{Izm}} \mathrm{s}$. Panels $\mathrm{c}-1$ and $\mathrm{c}-2$ show LA action potentials and $\mathrm{d} V / \mathrm{d} t_{\max }$ recorded from $S \mathrm{SR}_{\mathrm{Izm}} \mathrm{s}$ treated with olmesartan. The LA action potentials in panels a-1, b-1 and c-1 were recorded in normal Tyrode solution, and those in panels $\mathrm{a}-2, \mathrm{~b}-2$ and $\mathrm{c}-2$ were recorded during superfusion with Ang II. These data show that ATs were prolonged when Ang II was added to the perfusate (panels b-2 and c-2). AT was longer in untreated $\mathrm{SHR}_{\mathrm{Izm}} \mathrm{s}$ (panel b-2) than in preparations treated with olmesartan (panel c-2). The reductions in $\mathrm{d} V / \mathrm{d} t_{\max }$ during RES were small under Ang II. Thus, Ang II induced AT prolongation during RES in untreated $\mathrm{SHR}_{\mathrm{Izm}} \mathrm{S}$, which was antagonized by previous administration of olmesartan. The percent changes in atrial action potential parameters after RES in $\mathrm{SHR}_{\mathrm{Izm}} \mathrm{S}$ with and without olmesartan and/or Ang II are shown in Figure 3a. No significant differences were found among these groups except in AT. The percent changes in AT $(\triangle \mathrm{AT})$ before and after RES (Figure $3 \mathrm{~b}$ ) show that only the $\Delta \mathrm{AT}$ measured from untreated $\mathrm{SHR}_{\mathrm{Izm}} \mathrm{S}$ was significantly longer with Ang II compared with that measured from the other groups, which was similar to what is shown in Figure 2. Furthermore, Ang II induced conduction blocks during RES in untreated $\mathrm{SHR}_{\mathrm{Izm}} \mathrm{s}$ (Figures $4 \mathrm{a}$ and $\mathrm{b}$ ), and this occurred during the

Table 1 Systolic blood pressure, HR and cellular electrical properties from left atrial preparations from SHR $_{\text {Izm }}$

\begin{tabular}{|c|c|c|c|c|c|c|c|}
\hline & $B W(g)$ & $S B P(m m H g)$ & $H R$ (b.p.m.) & $R M P(m V)$ & $A P D_{75 \%}(m s)$ & $d \mathrm{~N} / d \mathrm{t}_{\max }\left(V s^{-1}\right)$ & $E R P(m s)$ \\
\hline OLM(-) & $436.0 \pm 27.1$ & $187.1 \pm 8.6$ & $335.5 \pm 20.9$ & $-78.9 \pm 3.3(-79.2 \pm 5.3)$ & $28.9 \pm 1.6(27.4 \pm 3.4)$ & $168.3 \pm 10.2(165.8 \pm 13.6)$ & $33.2 \pm 2.9(31.6 \pm 2.2)$ \\
\hline OLM(+) & $422.9 \pm 32.1$ & $* 164.7 \pm 7.6$ & $350.2 \pm 11.6$ & $-79.4 \pm 2.9(-80.5 \pm 4.1)$ & $27.6 \pm 3.2(27.0 \pm 2.0)$ & $170.3 \pm 13.8(166.8 \pm 16.7)$ & $34.5 \pm 2.2(34.4 \pm 1.8)$ \\
\hline
\end{tabular}

Abbreviations: $\mathrm{APD}_{75}, 75 \%$ repolarization duration; b.p.m., beats per minute; BW, body weight; $\mathrm{d} V / \mathrm{d} t_{\max }$, maximum rate of rise in phase 0 ; ERP, effective refractory period; HR, heart rate; $\mathrm{OLM}(+)$, treated with olmesartan; OLM(-), no treatment; RMP, resting membrane potential; SBP, systolic blood pressure.

The values were measured from SHR $\mathrm{Izm}_{\mathrm{Im}}$ with and without olmesartan (OLM) for 8 weeks. The cellular electrophysiological parameters were measured at $2 \mathrm{~Hz}$ of driving rate and the values in parentheses were measured under superfusion of angiotensin II $\left(10^{-8} \mathrm{M}\right)$.

parentheses were measured under
${ }^{*}$ Significant difference of $<0.05$.
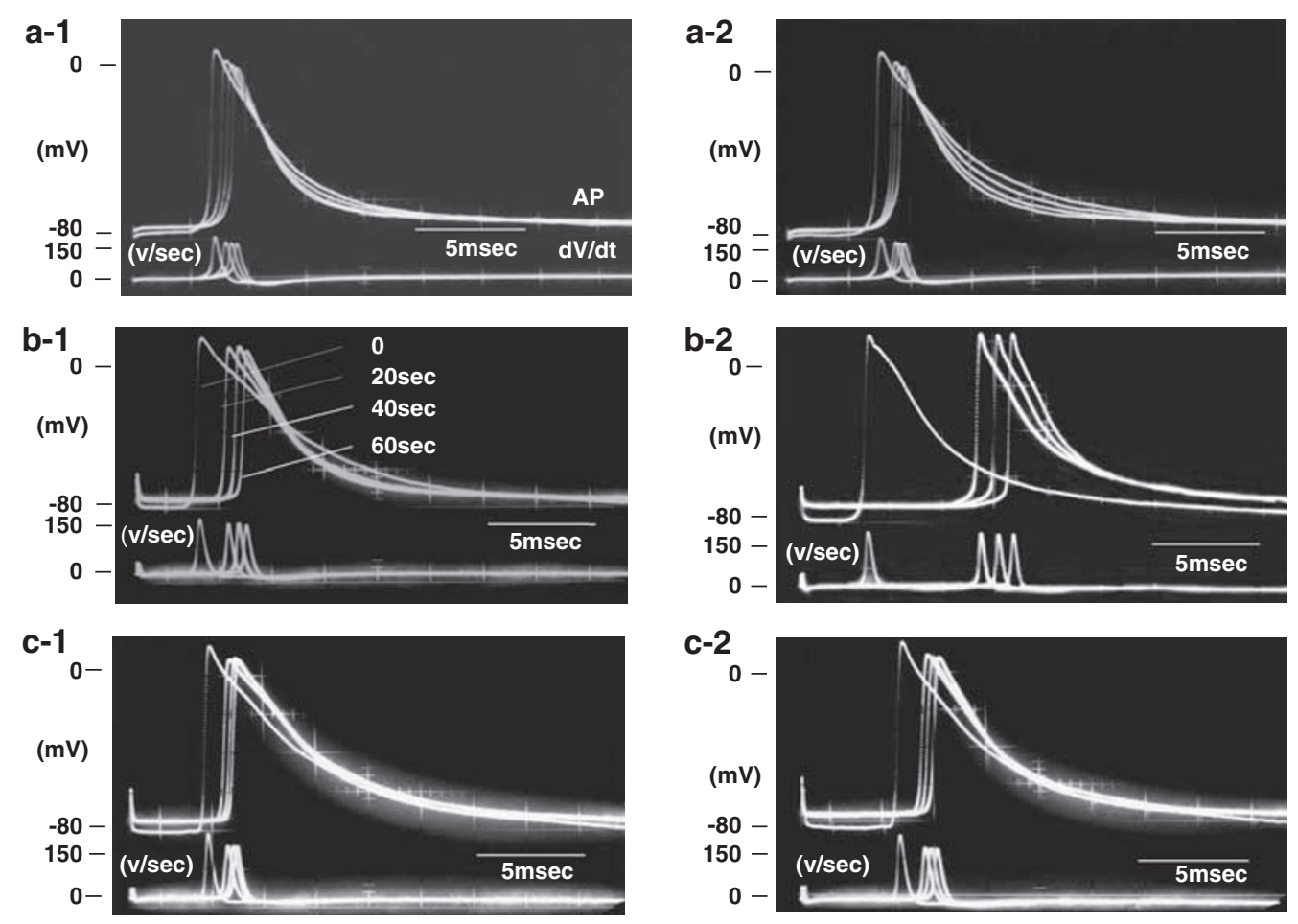

Figure 2 Representative change in atrial action potentials during rapid electrical stimulation. The photographs show the superimposed left atrial action potentials and $\mathrm{d} V / \mathrm{d} t$ from a WKY Izm (a-1 and a-2) and an SHR Izm (b-1, b-2 and c-1, c-2) during rapid electrical stimulation (RES) for 60 s. The four action potentials were recorded at 0, 20, 40 and 60 s after RES. Panels a-1, b-1 and c-1 show the recordings in normal Tyrode solution. Panels a-2, b-2 and c-2 show the recordings obtained in Tyrode solution containing angiotensin II. Middle panels b-1 and b- 2 show the action potentials from SHR $\mathrm{Izm}_{\mathrm{z}}$ without earlier administration of olmesartan. Lower panels $\mathrm{c}-1$ and $\mathrm{c}-2$ show action potentials from SHR $\mathrm{I}_{\mathrm{zm}} \mathrm{S}$ treated with olmesartan. In panel b-2, activation times were markedly prolonged in a preparation from an untreated $\mathrm{SHR}_{\mathrm{Izm}}$ superfused with angiotensin II. The activation times are slightly prolonged during angiotensin II perfusion in the preparation from a treated $\mathrm{SHR}_{\mathrm{Izm}}$ (panel c-2). AP, atrial action potential; dV/dt, rate of rise of action potential (unit; $\mathrm{s}^{-1}$ ); $\mathrm{SHR}$, spontaneously hypertensive rat. 


\begin{tabular}{|c|c|c|c|c|}
\hline & & (\%) RMP & $(\%) \mathrm{APD}_{75}$ & $(\%) \mathrm{dV} / \mathrm{dt}_{\max }$ \\
\hline \multirow{2}{*}{ OLM (-) } & Ang II (-) & $86.6 \pm 1.2$ & $86.7 \pm 2.1$ & $92.4 \pm 0.3$ \\
\hline & Ang II (+) & $87.6 \pm 1.5$ & $86.1 \pm 0.7$ & $91.7 \pm 0.3$ \\
\hline \multirow[t]{2}{*}{ OLM (+) } & Ang II (-) & $86.9 \pm 1.0$ & $86.6 \pm 1.2$ & $92.0 \pm 0.2$ \\
\hline & Ang II (+) & $87.2 \pm 1.1$ & $87.6 \pm 2.3$ & $91.8 \pm 0.6$ \\
\hline
\end{tabular}

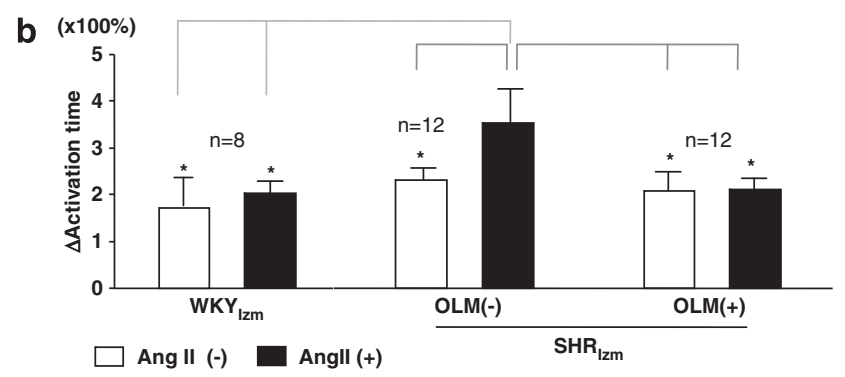

Figure 3 Atrial action potential parameters and changes in activation times before and after rapid electrical stimulation (RES). (a) Percent changes in atrial action potential parameters before and after RES for $60 \mathrm{~s}$ with or without olmesartan. No significant differences were found. In (b), percent changes in activation times ( $\triangle \mathrm{ATS}$ ) relative to the baseline value before RES are shown. Black columns indicate $\Delta \mathrm{AT}$ values with angiotensin II perfusion, and white columns show $\triangle A T$ values in normal Tyrode solution. Both sets of values were recorded $60 \mathrm{~s}$ after RES. Asterisks indicate a significant difference $(P<0.05)$. RMP, resting membrane potential; $\mathrm{APD}_{75}, 75 \%$ repolarization duration; $\mathrm{d} V / \mathrm{d} t_{\max }$, maximum rate of rise in phase 0 ; $A T$, activation time; OLM(-), without treatment with olmesartan; OLM(+), earlier administration of olmesartan; Ang $\mathrm{II}(+)$, angiotensin $\mathrm{II}$ added to the perfusate; Ang II(-), no angiotensin II in the perfusate; WKY, Wister Kyoto rat; SHR, spontaneously hypertensive rat.

latter half of RES. The transition from 1:1 to 2:1 conduction is seen from points 3 to 4 in the middle tracing of panel a in Figure 4. Panel b shows five still pictures captured from the DVD movie. The numbers on each picture correspond to the numbers in the middle tracing of panel a. In panels a and b, AT was gradually prolonged from points 2 to 3 , and the impulse propagation failed at $42 \mathrm{~s}$ after RES (Figure 4, panel b, picture 4). A Wenckebach type of block, as seen in panel b, was repeatedly observed at the end of RES and was accompanied by a slight change in $\mathrm{d} V / \mathrm{d} t_{\max }$. The conduction block occurrence rate during $60 \mathrm{~s}$ of RES was significantly greater with Ang II or with Ang II plus olmesartan than in the controls (Figure 4c). Thus, the conduction block under Ang II perfusion was suppressed by the AT1 receptor blocker, olmesartan.

Left atrial histological sections and the percentage of fibrous area in these sections are shown in Figure 5. In the upper panels, the interstitial spaces in the LA of untreated $\mathrm{SHR}_{\mathrm{Izm}} \mathrm{s}$ (Figure $5 \mathrm{a}$, middle panel, blue color) were larger than those in sections from the other treatments. The percentage fibrous area was significantly larger in untreated than in treated $\mathrm{SHR}_{\mathrm{Izm}} \mathrm{s}$ (Figure $5 \mathrm{~b}$ ).

\section{DISCUSSION}

\section{Reverse remodeling effects of Ang II type 1 receptor blocker}

A number of studies have used the rapid pacing model for investigating the relationship between atrial reverse remodeling and $\mathrm{AF}^{3,7,8}$ However, it is not fully understood whether Ang II influences the electrical properties of the remodeled left atrium. In our study, the prolongation of AT during RES, which was observed in $\mathrm{SHR}_{\mathrm{Izm}} \mathrm{s}$, was not found in $\mathrm{SHR}_{\mathrm{Izm}} \mathrm{S}$ treated with ARB (olmesartan), which may be related to the reverse remodeling action of ARB that prevents reentrant atrial arrhythmias. ${ }^{9,10}$ Chronic AT1 blockade with olmesartan attenuated the increased interstitial fibrous tissue in the left atrium of untreated $\mathrm{SHR}_{\mathrm{Izm}}$ s. Thus, our data showed that chronic AT1 blockade reduced interstitial fibrosis, but chronic AT1 blockade did not affect the LA action potential characteristics even during RES, except for AT. Therefore, the upregulation of AT1 receptors during RES may play a part in the onset of conduction blocking.

\section{Direct action of Ang II on the remodeled LA}

When the LA preparations from hypertensive animals were driven by RES, AT through the LA trabecula was lengthened, and conduction blocking appeared with Ang II perfusion (Figures 3 and 4). This Ang II-induced conduction block was antagonized by the previous administration of ARB (olmesartan) and the superfusion of ARB. The disappearance of Ang II-induced conduction abnormalities is not only because of the reverse LA remodeling action of ARB but also because of the direct action of ARB. This direct action of Ang II has not been previously reported. As the Ang II-induced conduction blocking was generated without a change in the action potential configuration, this phenomenon may be related to a reduction in gap junction conductance. In 1992, De Mello and Altieri ${ }^{11}$ used isolated ventricular cell pairs from adult rats and reported that Ang II reduced gap junction conductance by about 55\% within $20 \mathrm{~s}$, and that this effect was suppressed by the AT1 blocker losartan. This group suggested that this Ang II-induced action may depend on the activation of protein kinase C. A recent report showed that the AT1 blocker, olmesartan, normalized the prolonged intra-atrial conduction time after 4 weeks of rapid atrial pacing. ${ }^{7}$ Kistler et al. ${ }^{12}$ investigated the changes in atrial conduction delay and AF inducibility during infusion of Ang II into patients with supraventricular tachycardia who were undergoing curative ablation, and reported no acute effects of Ang II on these electrical parameters. Their data suggest that significant electrophysiological actions of Ang II may appear in some pathological conditions, such as tachycardia, ischemia and hypertension. Other possible data about a relationship between Ang II and atrial conduction in LA tissue have not been reported. ${ }^{3,5}$ Duffy and Wit ${ }^{13}$ strongly suggested that the remodeling of gap junctional cell coupling likely contributes to the occurrence of AF. Phosphorylation-dependent changes in gap junctions through AT1 activation seem to be related to Ang II-induced conduction blocking. ${ }^{14}$

\section{Clinical implications and limitations}

According to the hypothesis that AF is caused by a single source, AF could be driven by a single or small number of stable, discrete, highfrequency sources in the atrium. ${ }^{15}$ The Ang II-induced conduction block during RES observed in our study could promote the maintenance of AF. Therefore, the AT1 blocker olmesartan may be beneficial as a non-antiarrhythmic drug for AF because of its indirect and direct actions. ${ }^{16}$

There are some limitations to this study. The site of the conduction block was unclear, as AT includes both conductivity through trabeculae and tissue excitability from contact with the cathode-stimulating electrode. The space constant of mammalian heart tissue, wherein tissue excitability is critical for the onset of myocardial conduction, is known to be between 1 and $2 \mathrm{~mm}$. De Mello and Crespo ${ }^{17}$ isolated muscle trabeculae from rat right ventricles and studied the effect of Ang II on tissue excitability. They concluded that Ang II increased ventricular tissue excitability. Therefore, a major cause of Ang IIinduced conduction block could be detrimental conduction through trabeculae. The absence of a clear conduction sequence in whole preparations and also the absence of a detailed localization of interstitial fibrous tissue in trabeculae are additional limitations of this 
a
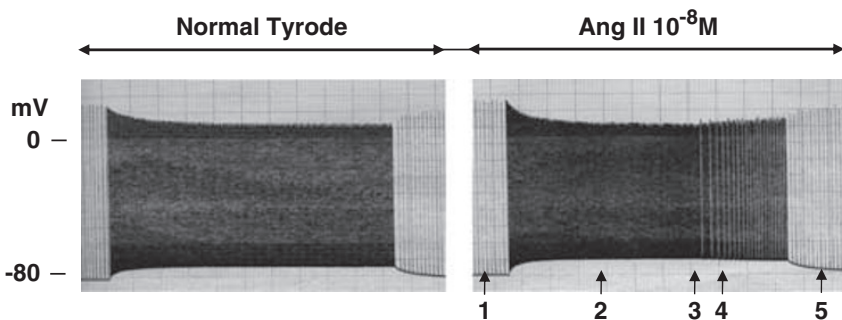

Ang $I 10^{-8} \mathrm{M}+$ OLM $10^{-7} \mathrm{M}$
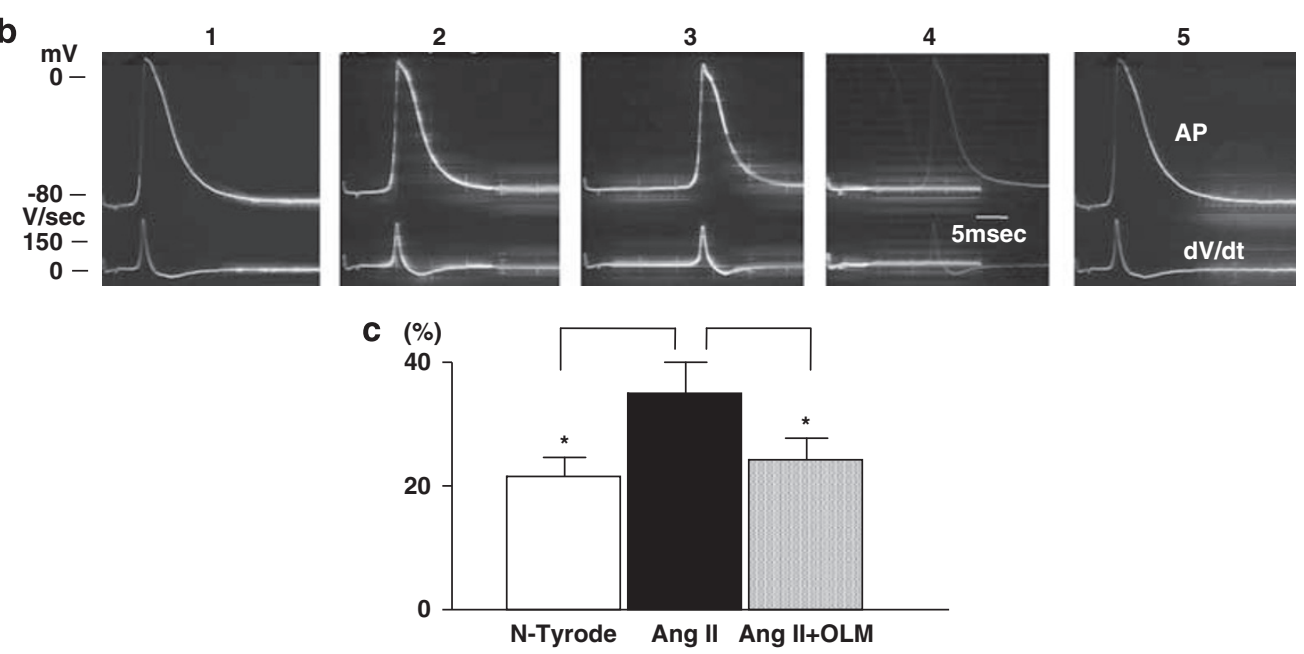

Figure 4 Angiotensin II induces conduction block during rapid electrical stimulation. (a) Consecutive action potential tracings during rapid electrical stimulation (RES) for $60 \mathrm{~s}$. The conduction blocks occurred under Ang II $\left(10^{-8} \mathrm{M}\right)$ perfusion as shown in the middle tracing. The transition from 1:1 to $2: 1$ conduction was seen between points 3 and 4 , as indicated by the arrows. The conduction block did not appear under angiotensin II plus olmesartan $\left(10^{-7} \mathrm{M}\right)$, as shown in the right tracing of (a). (b) Five frames captured from a DVD movie in which activation time was gradually prolonged from frame 1 to 3 and then blocked in frame 4. This Wenckebach type of block was repeatedly observed. In (c), the conduction block occurrence rates during RES for $60 \mathrm{~s}$ were significantly greater in the angiotensin II $(n=12)$ than in the control $(n=8)$ or the angiotensin II plus olmesartan $(n=12)$ groups. Ang II, angiotensin II; OLM, olmesartan; AP, atrial action potential; $\mathrm{d} V / \mathrm{d} t$, rate of rise of phase 0 . ${ }^{*} P<0.05$.

a

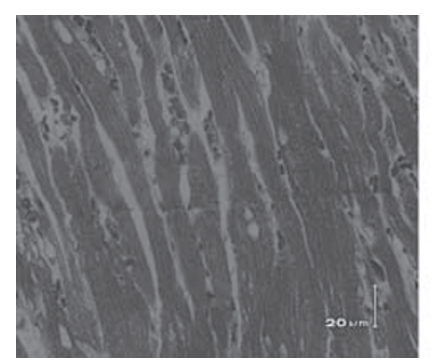

OLM(-)

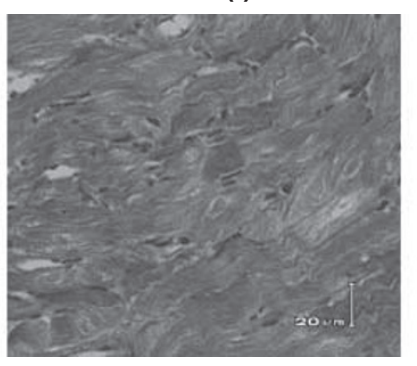

$\operatorname{OLM}(+)$

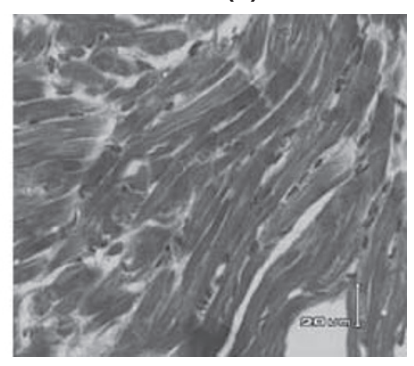

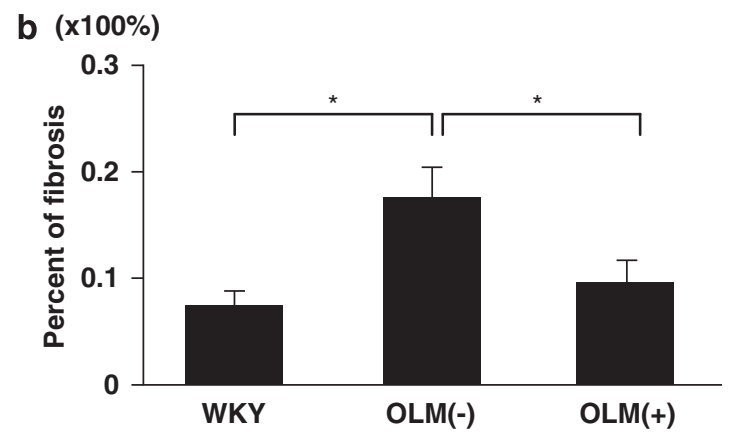

Figure 5 Histological sections of the left atrial wall and percent interstitial fibrous tissue area. Histological sections of the left atrial wall stained with Masson's trichrome are shown in (a). In (b), a quantitative analysis of the interstitial fibrous tissue area is expressed as the percent of fibrous area in a $100 \times 100 \mu \mathrm{m}^{2}$ area. OLM(-), untreated with olmesartan; OLM(+), treated with olmesartan. A full color version of this figure is available at the Hypertension Research journal online. 
study. It is difficult to provide an equal flow of a perfusate over an endocardial surface that is covered with intricate fine trabeculae, such as the preparations in this study. Thus, recording only impulse propagation through a thick trabecula in the center of the preparation was considered to be appropriate for this experiment.

\section{ACKNOWLEDGEMENTS}

We acknowledge Ms Yoshie Hirakawa for her excellent secretarial help.

1 Healey JS, Baranchuk A, Crystal E, Morillo CA, Garfinkle M, Yusuf S. Prevention of atrial fibrillation with angiotensin-converting enzyme inhibitors and angiotensin receptor blockers: a meta-analysis. J Am Coll Cardiol 2005; 45: 1832-1839.

2 Nattel S. Therapeutic implications of atrial fibrillation mechanisms: can mechanistic insights be used to improve AF management? Cardiovasc Res 2002; 54: 347-360.

3 Casaclang-Verzosa G, Gersh BJ, Tsang TS. Structural and functional remodeling of the left atrium: clinical and therapeutic implications for atrial fibrillation. J Am Coll Cardiol 2008; 51: 1-11.

4 Kumagai K. Upstream therapy for atrial fibrillation. Circ J 2007: (Suppl A): A75-A81.

5 Goette A, Lendeckel U. Nonchannel drug targets in atrial fibrillation. Pharmacol Ther 2004; 102: 17-36.

6 Brunner HR. The new oral angiotensin II antagonist olmesartan medoxomil: a concise overview. J Hum Hypertens 2002; 16(Suppl 2): S13-S16.

7 Nakashima H, Kumagai K. Reverse-remodeling effects of angiotensin II type 1 receptor blocker in a canine atrial fibrillation model. Circ J 2007; 71: 1977-1982.
8 Chrysostomakis SI, Karalis IK, Simantirakis EN, Koutsopoulos AV, Mavrakis HE, Chlouverakis GI, Vardas PE. Angiotensin II type 1 receptor inhibition is associated with reduced tachyarrhythmia-induced ventricular interstitial fibrosis in a goat atrial fibrillation model. Cardiovasc Drugs Ther 2007; 21: 357-365.

9 Burstein B, Nattel S. Atrial fibrosis: mechanisms and clinical relevance in atrial fibrillation. J Am Coll Cardiol 2008; 51: 802-809.

10 Varo N, Iraburu MJ, Varela M, López B, Etayo JC, Díez J. Chronic AT 1 blockade stimulates extracellular collagen type 1 degradation and reverses myocardial fibrosis in spontaneously hypertensive rats. Hypertension 2000; 35: 1197-1202.

11 De Mello W, Altieri P. The role of renin-angiotensin system in the control of cell communication in the heart: effects of enalapril and angiotensin II. J Cardiovasc Pharmacol 1992; 20: 643-651.

12 Kistler PM, Davidson NC, Sanders P, Fynn SP, Stevenson IH, Spence SJ, Vohra JK, Sparks PB, Kalman JM. Absence of acute effects of angiotensin II on atrial electrophysiology in humans. J Am Coll Cardiol 2005; 45: 154-156.

13 Duffy HS, Wit AL. Is there a role for remodeled connexins in AF? No simple answers. J Mol Cell Cardiol 2008; 44: 4-13.

14 Lampe PD, TenBroek EM, Burt JM, Johnson RG, Lau AF. Phosphorylation of connexin 43 on serine 368 by protein kinase $C$ regulates gap junctional communication. J Mol Cell Cardiol 2000; 149: 1503-1512.

15 Berenfeld O, Zaitsev AV, Mironov SF, Pertsov AM, Jalife J. Frequency-dependent breakdown of wave propagation into fibrillatory conduction across the pectinate muscle network in the isolated sheep right atrium. Circ Res 2002; 90: 1173-1180.

16 Murray KT, Mace LC, Yang Z. Nonantiarrhythmic drug therapy for atrial fibrillation. Heart Rhythm 2007; 4(3 Suppl): S88-S90.

17 De Mello WC, Crespo MJ. Cardiac refractoriness in rats is reduced by angiotensin II. J Cardiovasc Pharmacol 1995; 25: 5156 